\title{
Rotura espontánea del tendón extensor largo del pulgar - transferencia tendínea con técnica anestésica WALANT
}

\section{Spontaneous Rupture of Thumb Extensor Pollicis Longus - Tendon Transfer under the WALANT Anesthetics Technique}

\author{
Miguel Sanhueza Faúndez ${ }^{1}$ Camila Azócar Sanhueza ${ }^{1,2}$ Gabriel Carrasco Penna ${ }^{1}$ \\ ${ }^{1}$ Servicio de Ortopedia y Traumatología, Clínica Santa María, \\ Santiago, Chile \\ ${ }^{2}$ Departamento de Ortopedia y Traumatología, Universidad de Chile \\ Address for correspondence Miguel Sanhueza Faúndez, MD, Servicio de \\ Ortopedia y Traumatología, Clínica Santa María, Santa María 0500, \\ Providencia, Santiago, Chile (e-mail: migsanhuezaf@gmail.com).
}

Rev Chil Ortop Traumatol 2017;58:71-75.

\section{Resumen \\ Palabras Clave \\ - cirugía de la mano \\ - ELP \\ - extensor largo del pulgar \\ - transferencias tendíneas \\ - WALANT}

La rotura espontanea del tendón extensor largo del pulgar (ELP), es una patología infrecuente existiendo casos reportados en la literatura donde no se logra encontrar factores predisponentes. El manejo quirúrgico suele realizarse utilizando una técnica de transposición tendínea del tendón del extensor propio del índice. En la actualidad, la técnica anestésica de WALANT "Wide Awake Local Anesthesia with No-Torniquet" ha sido de amplio desarrollo en la cirugía de la mano, sobre todo para la resolución quirúrgica de patología de tendones, con buenos y excelentes resultados. Se presenta un caso de un paciente con rotura espontánea del tendón ELP, que fue manejado con una transferencia tendínea utilizando la técnica anestésica WALANT.

The spontaneous rupture of thumb extensor pollicis longus (EPL) is a rare disease with just a few case reports known where no trigger factor has been found. The surgical management is done by the proper index extensor tendon transposition. Nowadays, the WALANT anesthetic technique ("Wide Awake Local Anesthesia with NoTorniquet") has had a broad development in hand surgery, especially in surgery for tendon injuries, with good and excellent results. A clinical case is shown with a patient who had a spontaneous EPL rupture which was managed with a tendon transfer under the WALANT anesthetics technique.

\section{Introducción}

La rotura espontanea del tendón extensor largo del pulgar (ELP), es una patología infrecuente dentro de la patología de la mano. En la literatura han sido reportados distintos factores asociados dentro de los cuales los más frecuentes son la fractura de radio distal con una incidencia de hasta un $5 \%$ de las fracturas, la artritis reumatoide y la inyección

received

March 14, 2017

accepted

July 31, 2017

published online

August 23, 2017 sistémica o local de corticoides. ${ }^{1-4}$ Las actividades repetitivas con la extremidad, como son algunos tipos de danza y oficios, también han sido consideradas factores de riesgo para el desarrollo de esta patología. ${ }^{5-7}$ Otro factor asociado es la tendinosis en la zona del tubérculo de Lister debido a un efecto compresivo, donde incluso se ha planteado la descompresión profiláctica. ${ }^{8}$

Copyright (e) 2017 by Thieme Revinter

Publicações Ltda, Rio de Janeiro, Brazil

\section{License terms}

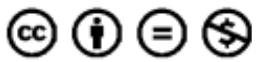


Si bien en la mayoría de los casos se puede identificar algún factor predisponente, también existe en la literatura el reporte de casos donde no se puede asociar ninguna causa a la rotura espontánea del tendón ELP. ${ }^{4}$

El manejo quirúrgico de una rotura espontanea es principalmente la transferencia tendinosa con el extensor propio del índice (EPI) y en segundo lugar, la realización de un injerto, generalmente de palmaris longus ipsilateral.

Para la realización de esa transferencia tendínea, y otras técnicas quirúrgicas dentro de la cirugía de la mano, en los últimos años ha sido de amplio desarrollo la utilización de la técnica anestésica WALANT "Wide Awake Local Anesthesia with No-Torniquet". ${ }^{9}$ Esa técnica consiste en la aplicación de un anestésico local (lidocaína) y un vasoconstrictor (epinefrina) para realizar la cirugía, sin necesidad de otro tipo de anestesia ni de torniquete braquial $\mathrm{y}$ ha tenido buenos resultados publicados en la literatura internacional. ${ }^{9-12}$ Permite disminuir los tiempos preoperatorios, no requiere de sedación $\mathrm{y}$, sobre todo, dado que el paciente está despierto, permite evaluar en el intraoperatorio la reconstrucción o transferencia tendínea con movimientos activos del paciente, lo que ayuda al cirujano a indicar con seguridad una rehabilitación precoz. ${ }^{10-12}$

Presentamos el caso de un paciente con rotura espontánea del tendón ELP que fue manejado con una transferencia tendínea aplicando la técnica anestésica WALANT.

\section{Caso Clínico}

Paciente de sexo masculino de 42 años de edad, diestro, sin antecedentes médicos ni quirúrgicos, tabaquismo activo de
3 cigarros a la semana y consumidor social de alcohol, ingeniero comercial con trabajo de oficina. Consulta por presentar dolor en región dorso-radial de tercio distal de antebrazo derecho asociado al movimiento de la muñeca, sin antecedentes de traumatismo. La radiografía de muñeca no muestra lesiones óseas agudas ni deformidades evidentes ( - Fig. 1).

Se diagnostica inicialmente una sinovitis de muñeca por sobreuso, se indicó analgesia con antiinflamatorios no esteroidales e inmovilizador de muñeca por un periodo de 7 días.

El paciente vuelve a consultar en 2 oportunidades por persistir la sintomatología. A la tercera semana de evolución es evaluado nuevamente por dolor y refiere no poder extender su pulgar. Al examen físico destaca un aumento de volumen radiocarpiano con dolor localizado en la zona radial de muñeca y ausencia completa de actividad del ELP. Ecografía de partes blandas de muñeca solicitada previamente demuestra el engrosamiento de los tendones extensores de segundo y tercer compartimento extensor con distensión de vainas sinoviales y aumento del líquido peritendíneo (-Fig. 2).

Se solicita resonancia magnética de muñeca que muestra pérdida de continuidad del ELP a nivel de la intersección con el segundo compartimento, una tenosinovitis del segundo y tercer compartimento extensor y escasa sinovitis radiocarpiana (-Fig. $\mathbf{3}$ ).

Se indica cirugía de transposición tendínea al tendón propio del índice la cual se realiza 6 semanas posteriores al inicio de la sintomatología del paciente.

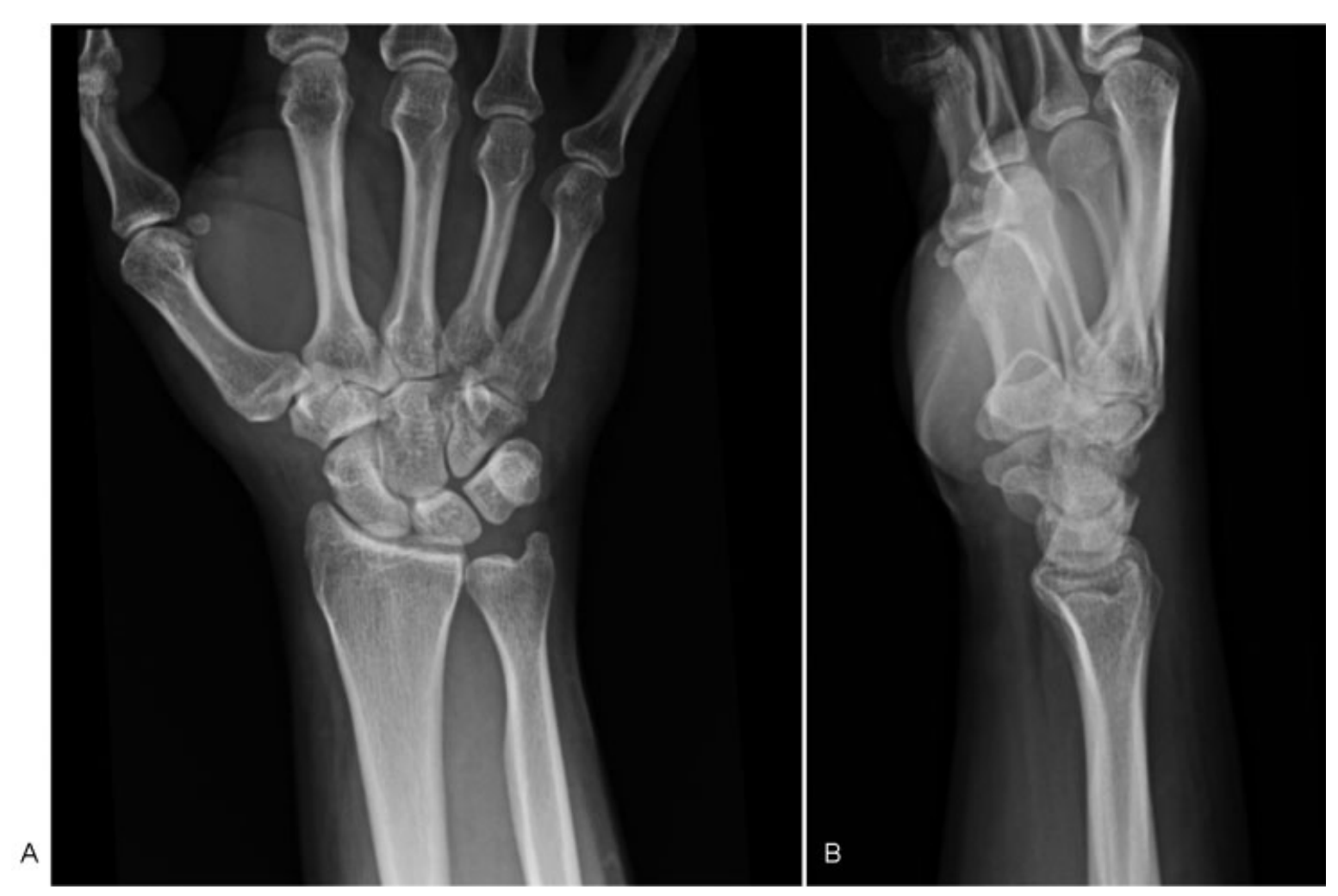

Fig. 1 Radiografía AP (A) y L (B) de la Muñeca Derecha. Radiografía se considera normal, sin encontrarse alteraciones anatómicas ni deformidades que pudiesen favorecer la rotura del ELP. 

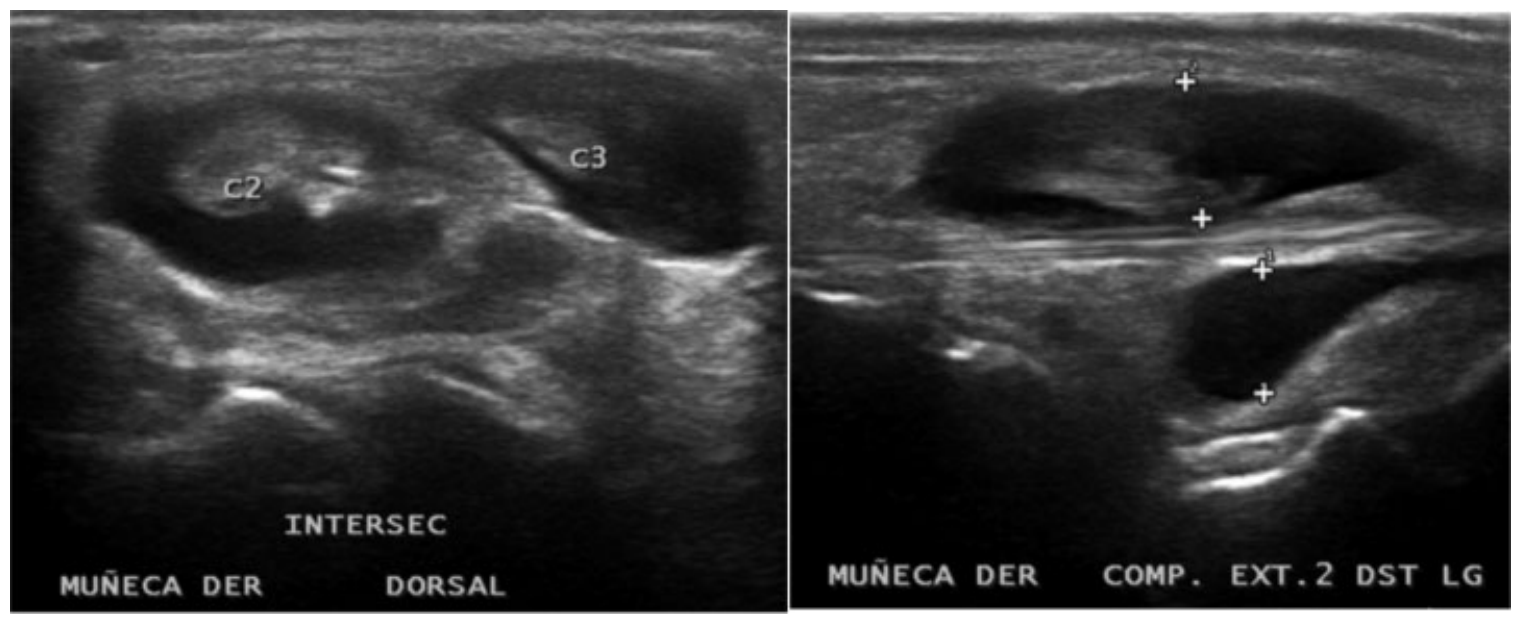

Fig. 2 Ecografía de Partes Blandas de la Muñeca Derecha. Se describe engrosamiento de tendones extensores de segundo y tercer compartimento extensor con distensión de vainas sinoviales y aumento del líquido peritendíneo.

\section{Técnica Quirúrgica}

En el pabellón central, paciente en decúbito supino en mesa quirúrgica y mesa lateral de mano, sin torniquete. Se realiza inyección de una mezcla de anestésico local (10cc de lidocaína al $2 \%$ ), vasoconstrictor (1cc de epinefrina en dilución de 1 / 10000 ) en zonas de abordaje según la técnica descrita por Lalonde. ${ }^{9,13}$ El paciente permanece despierto sin sedación en todo momento durante la cirugía.

Se realizan incisiones sobre la articulación metacarpofalángica del índice y sobre el tercer compartimento extensor logrando identificar el tendón EPI. Se realiza una tercera

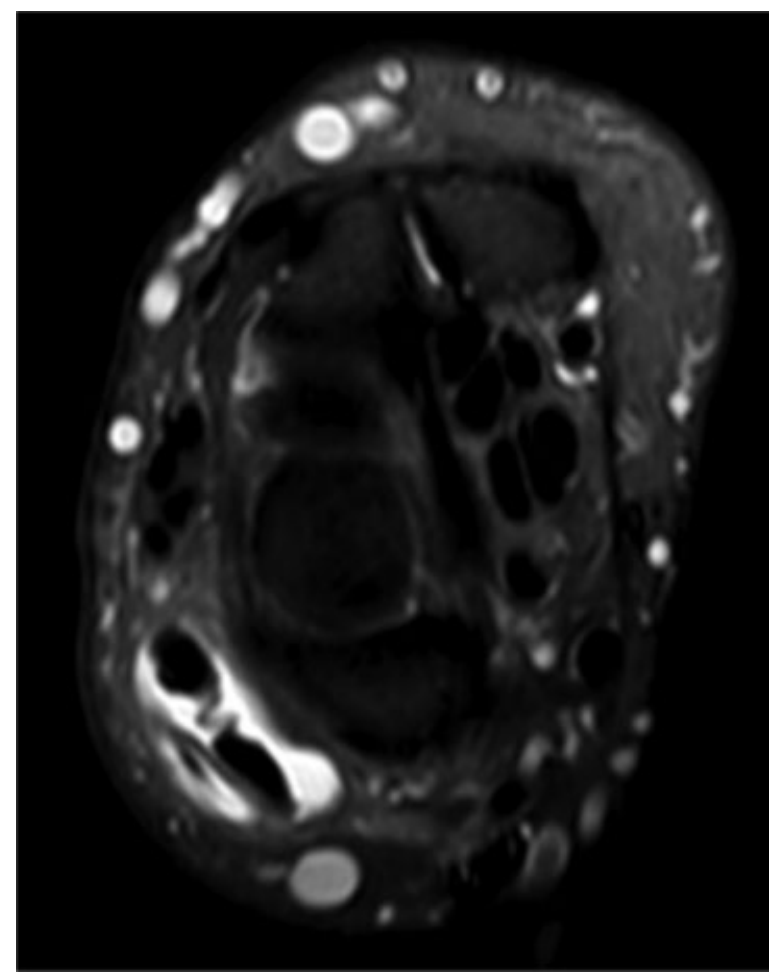

Fig. 3 Resonancia Magnética de la Muñeca Derecha. Se describe con tenosinovitis del segundo y tercer compartimento extensor. incisión sobre el primer metacarpiano identificándose el cabo distal del tendón ELP. Se realiza la sección del tendón EPI en la región distal y se traspasa por el subcutáneo hasta la zona del retináculo y luego al extremo distal del ELP donde se realiza una sutura tipo Pulvertaft ( - Fig. 4).

En el intraoperatorio se solicita al paciente realizar movimientos de flexo extensión del pulgar para evaluar la tensión de la sutura. El paciente es capaz de realizar esa indicación sin dificultad e incluso puede observar el resultado de su cirugía (-Fig. 5).

Se finaliza la cirugía con aseo y cierre de incisiones cutáneas y se deja con valva por 2 semanas y luego 2 semanas con inmovilizador removible del pulgar.

Control a las 4 semanas del postoperatorio se retira inmovilizador y se comprueba extensión activa del pulgar (-Fig. 6). Se indica entonces la rehabilitación Kinésica.

Se controla el paciente posteriormente con buena evolución, sin dolor y con extensión completa del pulgar contra-resistencia. Control al año desde la cirugía, el paciente se mantiene asintomático con función completa del pulgar.

\section{Discusión}

La rotura del tendón ELP se puede asociar a fracturas del extremo distal del radio, presencia de artritis reumatoide e infiltración tanto local como sistémica de corticoides. ${ }^{1-4}$ Factores asociados menos frecuentes se han descrito en la literatura y que en general hacen referencia a una actividad repetitiva de la extremidad lo que podría producir inflamación persistente y eventual rotura del tendón., $5,6,14$ Se ha descrito que la zona del tendón que está en contacto con el tubérculo de Lister es una región poco vascularizada lo que puede predisponer a una rotura en esa zona frente a compresiones o inflamación mantenida. ${ }^{15}$

El caso que se presenta es de una rotura espontánea del ELP sin un antecedente traumático, en un paciente sin antecedentes médicos ni de uso de corticoides. No refiere actividades repetitivas con su extremidad y tiene una radiografía que muestra una anatomía ósea normal sin 


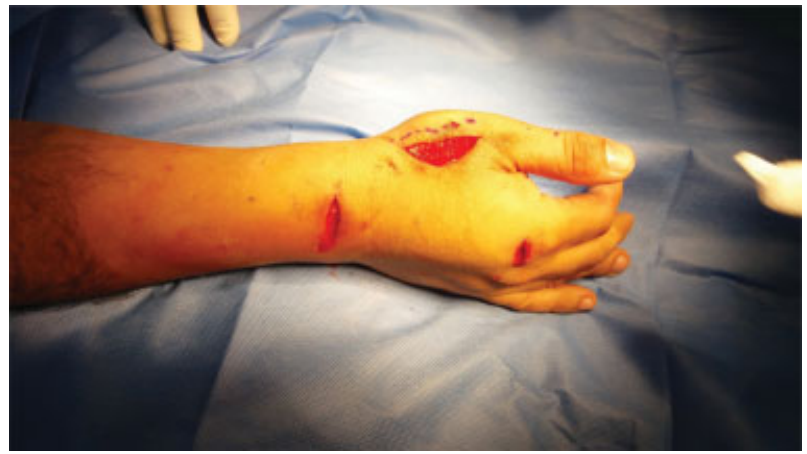

Fig. 4 Cirugía de Transposición del Tendón Extensor Propio del Índice al Tendón Extensor Largo del Pulgar con Técnica WALANT.

deformidades en la cortical dorsal, factor asociado a la rotura espontánea. ${ }^{4}$

Tanto la sintomatología como la imagenología del paciente, apuntan a una inflamación difusa sin poder identificar la
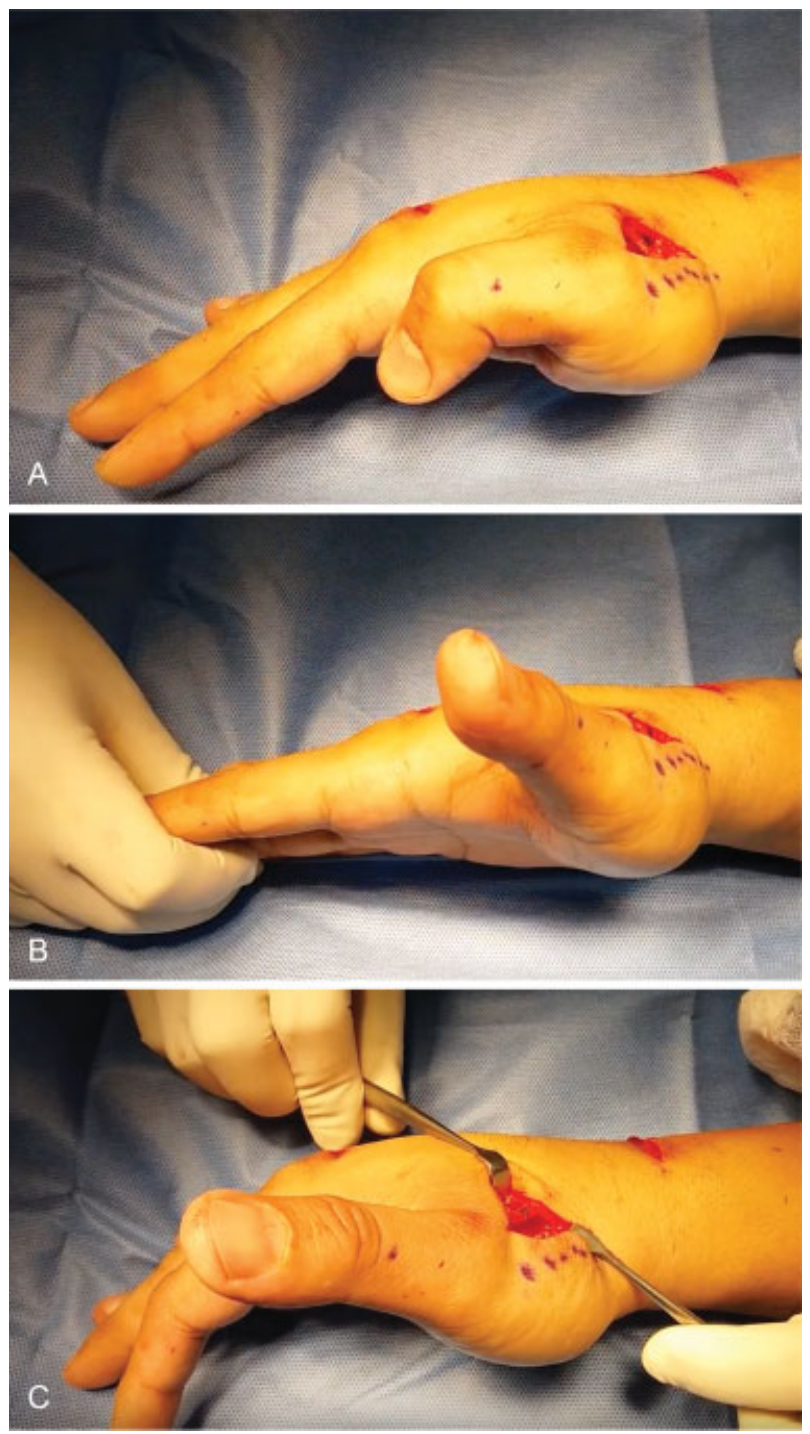

Fig. 5 Flexoextensión activa del pulgar en el intraoperatorio. (A). Flexión activa del pulgar. (B). Extensión activa del pulgar. (C). Se muestra la sutura mientras el paciente realiza extensión activa del pulgar).

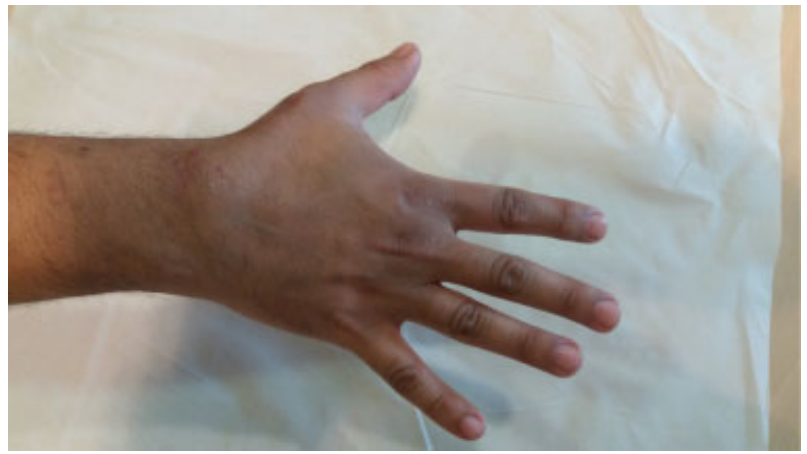

Fig. 6 Extensión activa a las 6 semanas.

causa específica de la rotura. Si bien no se realizó estudio serológico para descartar artritis reumatoide, el paciente no presentaba antecedentes de dolores articulares previos y su sintomatología actual era de reciente comienzo por lo que no apunta a una patología inflamatoria sistémica.

En las imágenes del paciente, se puede ver una inflamación de los tendones del segundo y tercer compartimento extensor en el período previo a la rotura del EPL. Se ha descrito que la presencia de una sinovitis mantenida produce riesgo de compresión del tendón ELP en la región hipovascular cercano al tubérculo de Lister y que eso podría ser el origen de una rotura del tendón. Ese podría ser el único factor de riesgo identificable en nuestro paciente y estaría involucrado en el desarrollo de la rotura. En nuestro paciente no se realizó estudio anatomopatológico de los cabos del tendón, lo cual podría haber aportado en la identificación de la causa de la lesión. En casos como esos, donde hay una sinovitis demostrada y mantenida, incluso se ha propuesto la liberación profiláctica del tendón para evitar la rotura completa. ${ }^{8}$ Esa es una hipótesis aún en estudio y que no es de regla en esos casos.

La transposición tendínea es el tratamiento de elección frente a esa patología, sobre todo cuando se trata de lesiones de mayor tiempo de evolución y en pacientes con artritis reumatoide con buenos resultados, buena satisfacción del paciente y bajas complicaciones. 8,16

En nuestro paciente se realiza la transposición tendínea bajo anestesia local con vasoconstricción sin torniquete utilizando la técnica de WALANT. Esa técnica anestésica está siendo ampliamente utilizada a nivel internacional en la cirugía de la mano y sobre todo para patología tendínea con buenos resultados y escasas complicaciones. ${ }^{16-18}$ Con la aplicación de WALANT, se ha descrito una disminución en los tiempos de estadía hospitalaria con respecto a técnicas de anestesia tradicionales que requieren sedación, y menor necesidad de solicitud de exámenes preoperatorios, sobre todo en pacientes jóvenes sin comorbilidad. ${ }^{10}$ Además, se ha descrito menor nivel de ansiedad preoperatoria y menor requerimiento de medicamentos analgésicos en el postoperatorio que en pacientes con anestesia tradicional. ${ }^{10}$

Dentro de las posibles complicaciones relacionadas a la técnica de anestesia tipo WALANT, la más temida es la necrosis del dedo debido a la vasoconstricción basados en 
los estudios de inicios del siglo $\mathrm{XX}$, donde se reportan casos con esa complicación por el uso específico de Procaína. Eso ha sido desestimado en las últimas revisiones donde no se han encontrado casos de necrosis posterior a la anestesia de WALANT en revisiones con más de 2000 pacientes. ${ }^{9,19}$

Específicamente se prefiere esa técnica en la cirugía de transferencia tendínea puesto que permite comprobar en el intraoperatorio la tensión de la sutura y permite su corrección en caso de ser necesaria ${ }^{18}$ para lograr una completa e inmediata movilización activa de flexoextensión. Eso le confiere seguridad tanto al paciente como al cirujano con respecto a la rehabilitación precoz. También se describen buenos resultados con respecto a la satisfacción del paciente frente a su experiencia en el intraoperatorio, dado que se siente partícipe del resultado quirúrgico y confiado frente a la rehabilitación. ${ }^{13,17-20}$

En nuestro paciente destacamos la participación activa durante la cirugía y durante su rehabilitación, lo que permitió un retiro precoz de la inmovilización rígida y una movilización activa completa a la cuarta semana de post operado.

\section{Conclusión}

La rotura espontánea del tendón extensor largo del pulgar es una patología infrecuente y generalmente asociada a factores predisponentes los cuales hay que descartar. Se presenta un caso de rotura espontánea del tendón ELP sin causa identificable. La transferencia al tendón extensor propio del índice es la técnica elegida por los autores para el tratamiento de esa lesión donde la técnica anestésica WALANT proporciona buenos resultados de satisfacción del paciente y permite comprobar el éxito de la sutura en el intraoperatorio permitiendo una rehabilitación precoz y segura. Proponemos el uso de la técnica anestésica de WALANT para la cirugía tendínea de la mano, como una técnica confiable, segura y reproducible, con beneficios evidentes sobre todo en tenorrafias comparado con las técnicas anestésicas que requieren sedación.

\section{Responsabilidades Éticas}

Protección de personas y animales. Los autores declaran que para esta investigación no se han realizado experimentos en seres humanos ni en animales.

Confidencialidad de los datos. Los autores declaran que han secundado los protocolos de su centro de trabajo sobre la publicación de los datos de los pacientes.

Derecho a la privacidad y consentimiento informado. Los autores han obtenido el consentimiento informado de los pacientes y/o sujetos referidos en el artículo. Este documento obra en poder del autor de correspondencia.
Conflicto de Interés

Los autores declaran que no existen conflictos de interés.

\section{Referencias}

1 Cigna E, Özkan Ö, Mardini S, Chiang PT, Yang CH, Chen HC. Late spontaneous rupture of the extensor pollicis longus tendon after corticosteroid injection for flexor tenosynovitis. Eur Rev Med Pharmacol Sci 2013;17(06):845-848

$2 \mathrm{Kim} \mathrm{CH}$. Spontaneous rupture of the extensor pollicis longus tendon. Arch Plast Surg 2012;39(06):680-682

3 Rada EM, Shridharani SM, Lifchez SD. Spontaneous atraumatic extensor pollicis longus rupture in the nonrheumatoid population. Eplasty 2013;13:e11

$4 \mathrm{Hu}$ C-H, Fufa D, Hsu C-C, Lin Y-T, Lin C-H. Revisiting spontaneous rupture of the extensor pollicis longus tendon: eight cases without identifiable predisposing factor. Hand (NY) 2015;10(04):726-731

5 Jain A, Goyal N, Mishra P. Spontaneous rupture of EPL and ECRB tendons in a washerwoman: an unusual phenomenon. Hand Surg 2014;19(02):241-244

6 Shifflett GD, Ek ET, Weiland AJ. Spontaneous rupture of the extensor pollicis longus in a break-dancer. Eplasty 2014;14:e4

7 Navaratnam AV, Ball S, Eckersley R. Prophylactic decompression of extensor pollicis longus to prevent rupture. BMJ Case Rep 2013;2013(13):bcr2013010196

8 Diep GK, Adams JE. The Prodrome of Extensor Pollicis Longus Tendonitis and Rupture: Rupture May Be Preventable. Orthopedics 2016;39(05):318-322

9 Lalonde DH. Reconstruction of the hand with wide awake surgery. Clin Plast Surg 2011;38(04):761-769

10 Davison PG, Cobb T, Lalonde DH. The patient's perspective on carpal tunnel surgery related to the type of anesthesia: a prospective cohort study. Hand (NY) 2013;8(01):47-53

11 Farhangkhoee $\mathrm{H}$, Lalonde J, Lalonde DH. Wide-awake trapeziectomy: video detailing local anesthetic injection and surgery. Hand (NY) 2011;6(04):466-467

12 Higgins A, Lalonde DH, Bell M, McKee D, Lalonde JF. Avoiding flexor tendon repair rupture with intraoperative total active movement examination. Plast Reconstr Surg 2010;126(03):941-945

13 Lalonde DH. Wide-awake extensor indicis proprius to extensor pollicis longus tendon transfer. J Hand Surg Am 2014;39(11): 2297-2299

14 Choi JC, Kim WS, Na HY, et al. Spontaneous rupture of the extensor pollicis longus tendon in a tailor. Clin Orthop Surg 2011;3(02):167-169

15 Engkvist O, Lundborg G. Rupture of the extensor pollicis longus tendon after fracture of the lower end of the radius-a clinical and microangiographic study. Hand 1979;11(01):76-86

16 O'Sullivan MB, Singh H, Wolf JM. Tendon Transfers in the Rheumatoid Hand for Reconstruction. Hand Clin 2016;32(03):407-416

17 Lalonde D, Eaton C, Amadio P, Jupiter J. Wide-awake Hand and Wrist Surgery: A New Horizon in Outpatient Surgery. Instr Course Lect 2015;64:249-259

18 Tang JB. Wide-Awake Primary Flexor Tendon Repair, Tenolysis, and Tendon Transfer. Clin Orthop Surg 2015;7(03):275-281

19 Nodwell T, Lalonde D. How long does it take phentolamine to reverse adrenaline-induced vasoconstriction in the finger and hand? A prospective, randomized, blinded study: The Dalhousie project experimental phase. Can J Plast Surg 2003;11(04):187-190

20 Chowdhry S, Seidenstricker L, Cooney DS, Hazani R, Wilhelmi BJ. Do not use epinephrine in digital blocks: myth or truth? Part II. A retrospective review of 1111 cases. Plast Reconstr Surg 2010; 126(06):2031-2034 